

Cite this: Phys. Chem. Chem. Phys., $2016,18,16196$

Received 13th April 2016, Accepted 24th May 2016

DOI: $10.1039 / c 6 c p 02475 k$

www.rsc.org/pccp

\section{Flexibilities of isoindoline-derived spin labels for nucleic acids by orientation selective PELDOR $\dagger$}

\author{
N. Erlenbach, ${ }^{a}$ B. Endeward, ${ }^{a}$ P. Schöps, ${ }^{a}$ D. B. Gophane, ${ }^{b}$ S. Th. Sigurdsson ${ }^{b}$ and \\ T. F. Prisner*a
}

Pulsed electron electron double resonance experiments with rigid spin labels can reveal very detailed information about the structure and conformational flexibility of nucleic acid molecules. On the other hand, the analysis of such data is more involved the distance and orientation information encoded in the time domain data need to be extracted and separated. In this respect studies with different spin labels with variable internal mobility are interesting and can help to unambiguously interpret the EPR data. Here orientation selective multi-frequency/multi-field 4-pulse PELDOR/DEER experiments with three recently presented semi-rigid or conformationally unambiguous isoindoline-derived spin labels were performed and simulated quantitatively by taking the spin label dynamics into account. PELDOR measurements were performed for a 20-mer dsDNA with two spin labels attached to two defined uridine derivatives. Measurements were recorded for different spin label positions within the double helical strand and for different magnetic field strengths. The experimental data sets were compared with simulations, taking into account the previously described dsDNA dynamics and the internal motions of the spin label itself, which had shown distinct differences between the three spin labels used. The ExImU spin label shows a free rotation around a single bond, which averages out orientation effects, without influencing the distance distribution as it can occur in other spin labels. The ${ }^{I m} U$ and ${ }^{O x} U$ spin label, on the other hand, show distinct orientation behaviour with minimal intrinsic motion. We could quantitatively determine this internal motion and demonstrate that the conformational dynamics of the nucleic acid and the spin label can be well separated by this approach.

\section{Introduction}

Nucleic acids play a fundamental role in all forms of life as they are involved in storing, transmitting and expressing genetic information. Furthermore, they have various other functional roles, such as protein synthesis and regulation of gene expression. Information about nucleic acid structure and dynamics gives insight into how they are able to carry out their functions. Their structures, and to some extent dynamics, can be investigated with the established methods of X-ray crystallography ${ }^{1,2}$ and nuclear magnetic resonance (NMR) spectroscopy. ${ }^{3-6}$ However, for a better understanding of the functions of subtle nucleic acids dynamics, for example sequence dependent internal dynamics of duplexes involved with protein recognition, other

\footnotetext{
${ }^{a}$ Institute of Physical and Theoretical Chemistry, Center of Biomolecular Magnetic Resonance, Goethe University, Max-von-Laue-Str. 7, D-60438 Frankfurt am Main, Germany.E-mail: prisner@chemie.uni-frankfurt.de

${ }^{b}$ Department of Chemistry, Science Institute University of Iceland, Dunhaga 3, 107 Reykjavik, Iceland

$\dagger$ Electronic supplementary information (ESI) available: Rawdata for the Q- and G-band measurements. Additional comparisons between experimental and simulated data. See DOI: 10.1039/c6cp02475k
}

spectroscopic techniques, such as fluorescence-based techniques and electron paramagnetic resonance (EPR) spectroscopy are very useful. Both of these techniques require reporter groups, fluorescence spectroscopy requires fluorescent labels while EPR spectroscopy uses paramagnetic spin labels. When single labels are used, both techniques can give information about dynamics, but for structural studies, the distances between two labels must be measured, either using the Foerster resonance energy transfer ${ }^{7,8}$ (FRET) or pulsed electron-electron double resonance ${ }^{9-14}$ (PELDOR/DEER).

Commonly used reporter groups for EPR spectroscopy are aminoxyl radicals, usually called nitroxides. Nitroxides are relatively stable and can be synthetically manipulated and attached site specifically to nucleic acids, a technique referred to as sitedirected spin labeling ${ }^{15,16}$ (SDSL). Most nitroxide spin labels have some inherent mobility due to rotation around the tether between label and nucleic acid, giving rise to imprecision when used for distance measurements. We have developed the rigid spin labels Ç and $\mathrm{Çm}^{17,18}$ (Fig. 1a) to circumvent this drawback, in which the nitroxides are fused to a nucleobase and do, therefore, not have motion independent of the base. However, if the intrinsic motion of the spin label is strongly restricted, 
a)<smiles></smiles><smiles></smiles>

c)

$$
\begin{aligned}
& \text { Ç }(R=H) \\
& \text { Çm }\left(\mathrm{R}=\mathrm{OCH}_{3}\right)
\end{aligned}
$$

$\operatorname{Im} U \quad(X=N H)$

OXU $\quad(\mathrm{X}=0)$

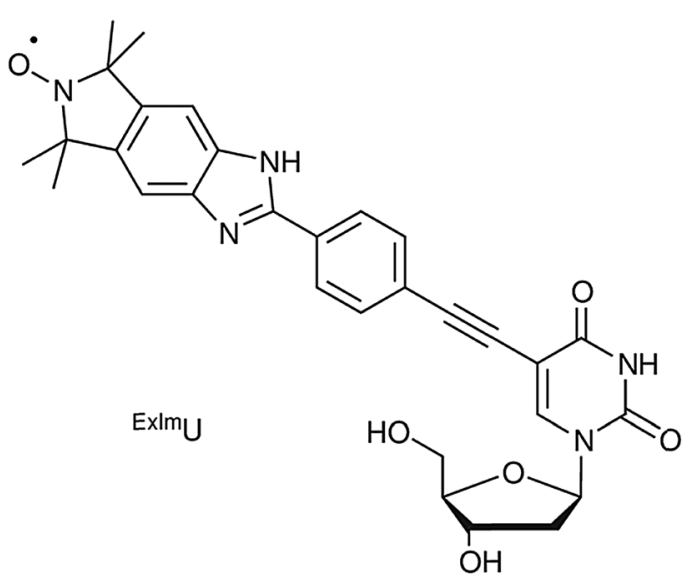

d)

\section{5'- GCX AGT GCG CAC GCG CGA TC - 3' 3'- CGA TCA CGC GXG CGC GCT AG - 5' $\operatorname{DNA}(1,9)$}

e)

\section{5'- GCX AGT CGC GCG CAC GCA TC - 3' 3'- CGA TCA GCG CGC GXG CGT AG - 5' $\operatorname{DNA}(1,12)$}

Fig. 1 Spin-labeled nucleosides. (a) Rigid spin labels Ç and Çm (b) Isoindoline derived spin labels ${ }^{I m} U$ and ${ }^{\circ x} U$. (c) Benzimidazoline spin label ExlmU (d and e) The sequence for DNA(1,9) and DNA(1,12),respectively. The $X$ indicates the spin label position. The internal motion of the DNA, found in previous studies, which shows a change in the radius of the DNA cylinder without any change of the helix pitch, was utilized along with a simple three axis model for the internal motion of the spin labels to simulate all PELDOR data sets simultaneously. ${ }^{21}$ Excellent fits of all experimental data could be obtained by assuming rotation of the spin labels around the molecular $x$-axis of the spin label, which is equal to the $\mathrm{N}-\mathrm{O}$ bond direction, with different amplitudes for the three spin labels investigated. Our analysis proves the semi-rigidity of the isoindoline spin label and gives quantitative values of the internal mobility of these labels. In the case of ${ }^{\prime m} U$, the rotation around the $\mathrm{N}-\mathrm{O}$ axis of the nitroxide which has the largest influence on the orientation dependence is around $\pm 5^{\circ}$, whereas for ${ }^{\circ} \mathrm{X} U$ it was found to be twice as high. In the case of the ExIm $U$ the assumption of a free rotation around the tether that reduces orientation effects could be deduced. This detailed analyses of the dynamics of ${ }^{I m} \cup,{ }^{O x} U$ and ${ }^{E x I m} U$ will facilitate the use of these spin labels for further studies of nucleic acid motifs. the common assumption of random orientation distribution, which applies to flexible labels, is no longer correct. Therefore, distance analysis of a single time trace using Tikhonov regularization is not applicable. If only reliable distance information are required, this would be a drawback of rigid spin labels. However, the large advantage of rigid spin labels is the access to information about the relative orientation between spin labels and flexibility of the system under study. ${ }^{19,20}$ We have shown that the analysis of distances and relative orientations between such labels using multi-frequency/multi-field PELDOR data sets can give information about the internal motion of double stranded $\mathrm{DNA}^{21}$ and the three-way junction of the cocaine aptamer, ${ }^{22}$ which would be inaccessible with flexible spin labels.

Although Ç shows a high orientation selection in PELDOR experiments, its preparation is non-trivial. For this reason we have recently introduced isoindoline spin labels that are linked by a single bond to a uridine. The semi-rigid spin labels ${ }^{\mathrm{Im}} U$ and ${ }^{\mathrm{Ox}} \mathrm{U}$ (Fig. 1b) are restricted in rotation around the single bond linking them to the nucleobase. ${ }^{23}$ In particular the ${ }^{\mathrm{Im}} \mathrm{U}$ spin label can form an intramolecular hydrogen bond between the $\mathrm{NH}$ of the benzimidazole and $\mathrm{O} 4$ of the nucleobase. Both ${ }^{\mathrm{Im}} \mathrm{U}$ and ${ }^{\mathrm{Ox}} \mathrm{U}$ show orientation-dependent changes in the PELDOR time traces as a function of the chosen probe and pump frequencies, although they are less rigid than Ç. Nevertheless even this small intrinsic motion could have an influence on the analysis of the dynamics of the nucleic acids. Therefore, it is necessary to gain an in depth knowledge about the mobility of the spin label itself to be able to separate this from the motion of the biopolymer.

In addition to ${ }^{\mathrm{Im}} \mathrm{U}$ and ${ }^{{ }^{O x}} \mathrm{U}$, we have prepared the "conformationally unambiguous spin label" ${ }^{, 24}$ ExIm $U$ (Fig. 1c), which can freely rotate around the single bonds flanking the acetylene. ${ }^{25}$ Since the $\mathrm{N}-\mathrm{O}$ bond of the nitroxide aligns to the same axis as these bonds, the displacement of the unpaired electron spin due to this rotation should be negligible. However, the orientation should be rather efficiently averaged out by the rotation and this label could be therefore used for direct distance determinations. It was confirmed that PELDOR of this label does not contain observable orientation information at Q-Band frequencies where such measurements are usually performed. ${ }^{26}$ Nevertheless at higher frequencies, where the in-ring orientations can be also distinguished, even ${ }^{{ }^{E x I m}} \mathrm{U}$ shows some retained orientation dependence (see below).

In this paper, we describe the detailed analysis of the internal motion of the spin labels ${ }^{\mathrm{Im}} \mathrm{U},{ }^{\mathrm{Ox}} \mathrm{U}$ and ${ }^{\mathrm{ExIm}} \mathrm{U}$. Two DNA duplexes $(\operatorname{DNA}(1,9)$ and $\operatorname{DNA}(1,12))$, which have already been analyzed with regards to their mobility using the rigid spin label Ç, were spin labeled with ${ }^{{ }^{I m}} U,{ }^{O x} U$ and ${ }^{E^{2} I m} U$ and measured by multifrequency/multi-field PELDOR.

\section{Experimental}

Six different samples were used to analyse the flexibility of isoindoline-derived spin labels. The spin labels, ${ }^{{ }^{I m}} \mathrm{U},{ }^{\mathrm{Ox}} \mathrm{U}$ and ${ }^{\mathrm{ExIm}} \mathrm{U}$, were incorporated pairwise into two DNA duplexes (Fig. 1). 
The samples were previously measured at X-band frequencies ${ }^{25}$ and for this work, they were additionally measured at Q-band (33.8 GHz/1.2 T) and G-band (180 GHz/6.4 T) frequencies. The dead-time-free four-pulse PELDOR sequence ${ }^{11}$ was used for all experiments. In order to extract the intramolecular PELDOR form factor the raw data were pre-processed using the DeerAnalysis2013 software package, ${ }^{27}$ with homogenous 3D background correction.

\subsection{PELDOR sample preparation}

The DNA samples for PELDOR measurement were prepared by annealing $10 \mathrm{nmol}$ of each strand with $10 \mathrm{nmol}$ of its complementary strand in phosphate buffer $(100 \mu \mathrm{L}, \mathrm{pH} 7.0, \mathrm{NaCl}$ (100 $\mathrm{mM})$, and EDTA $(0.1 \mathrm{mM}))$, followed by evaporation of the water. The annealed dried samples were subsequently dissolved in $20 \%$ ethylene glycol/ $\mathrm{H}_{2} \mathrm{O}(100 \mu \mathrm{L})$. The final spin concentrations for all samples are listed in the ESI. $\dagger$ All samples were rapidly frozen and stored in liquid nitrogen until they were measured.

\subsection{Q-band measurements}

A Bruker Elexsys E580 X/Q-band spectrometer equipped with an Oxford CF935 cryostat and a phase unlocked PELDOR frequency unit was used. A home built loop-gap Q-band resonator with a larger bandwidth was used to achieve higher offsets between pump and probe pulses. Microwave pulses were amplified by a $10 \mathrm{~W}$ solid state Q-band amplifier. $64 \mathrm{~ns}$ $(\pi / 2$ and $\pi)$-pulses were used for detection and a $34 \mathrm{~ns}(\pi)$ pump pulse was used. The delay between the first and second probe pulses was $132 \mathrm{~ns}$. The pulse separation between the second and third probe pulse was typically $2.0 \mu \mathrm{s}$. The frequency of the pump pulse was fixed to the intensity maximum of the nitroxide powder spectrum to obtain maximum pumping efficiency. The probe frequency was chosen to be 50, 100 or $150 \mathrm{MHz}$ below this frequency (Fig. 2). All experiments were carried out at a temperature of $50 \mathrm{~K}$.

\subsection{G-band measurements}

All G-band EPR experiments were performed with a home built G-band spectrometer equipped with two independent frequency sources. ${ }^{28-30}$ The probe pulse length were between 32-40 ns for the $\pi / 2$-pulse and $60-70 \mathrm{~ns}$ for the $\pi$-pulse and


Fig. 2 Simulated Field-sweep-spectra at (a) Q-band and (b) G-band frequencies indicating the positions of the PELDOR pump und detection pulses. Green arrows show the position of the pump pulses, the red arrows the detection pulses. the pump inversion pulse was adjusted to be about 60 ns. The pulse separation between the first and second probe pulse was $220 \mathrm{~ns}$ and $1.8 \mu \mathrm{s}$ between the second and third probe pulse. The repetition time of the experiment was between 6 and $8 \mathrm{~ms}$. All experiments were carried out at a temperature of $40 \mathrm{~K}$. Every set of experiments consisted of around 80 time traces averaged with 100 shots per point and were recorded at different field positions across the EPR spectrum, corresponding roughly to the $g_{x x}, g_{y y}$, and $g_{z z}$ positions of the spectrum (Fig. 2). The probe frequency was set in all cases at a constant offset of $60 \mathrm{MHz}$ above the pump frequency.

\subsection{Simulations}

A home-written Matlab@ program was used for analysis of the PELDOR data. After generating the positions and orientation of the two unpaired electron spins according to the conformational flexibility of both the helix and the spin label, PELDOR time traces were simulated and compared with experimental data. For every simulation, an ensemble of 500 conformers was calculated, to represent the conformational ensemble in the frozen solution. Each simulation was performed in two steps. First, the DNA helix conformational flexibility was calculated according to the correlated change of the helix radius and the length of the DNA, as described in Marko et al. ${ }^{21}$ These calculations were done with two constant helix parameters: the helix of the B form DNA was calculated with a $3.3 \mathrm{~nm}$ pitch and a contour length of $0.485 \mathrm{~nm}$, which presents the distance between two base pairs along the helix. The mean radius of the helix cylinder was set to $0.585 \mathrm{~nm}$, with a Gaussian distributed standard deviation of $0.065 \mathrm{~nm}$, which reveals a negative twist-stretch coupling. Each $\mathrm{C}^{\prime}{ }^{\prime}$ atom of the nucleosides was calculated with these parameters. In a second step, the electron spin position of the spin label and their mutual orientation was constructed from the helix. Two rotations are needed to obtain the angles describing their relative orientation. The first rotation started with the vector between the nucleoside C1a and $\mathrm{C} 1 \mathrm{~b}$ atom and goes around the $z$ axis with $\alpha=105^{\circ}$. Additionally a rotation by $\beta=10^{\circ}$ around the normal vector of the plane between the $z$ axis and the new vector was applied (Fig. 3). The resulting vector starts from the $\mathrm{C} 1 \mathrm{a}$-atom in the direction of the electron spin. As the distance between the C1a and the electron

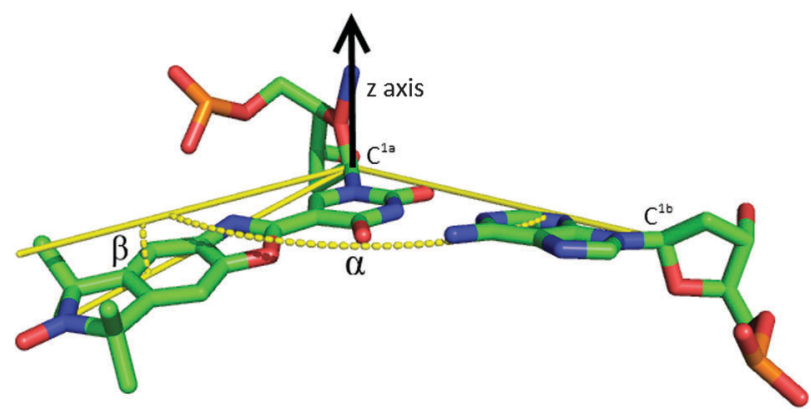

Fig. 3 Isoindoline-derived spin label paired to Adenine. The angles between the yellow lines define the rotation angles to calculated the electron spin position with the start C1 coordinates of the paired nucleobases. 


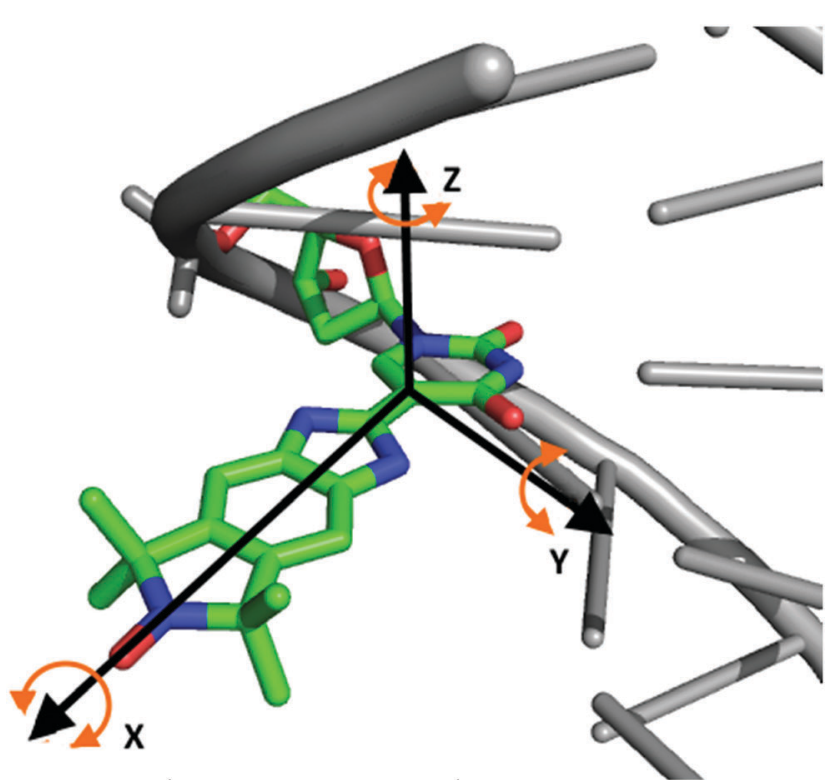

Fig. 4 Modes of spin label motions shown for the ImU-labelled duplex DNA. In addition to the DNA twist-stretch motions, ${ }^{9}$ rotational variations around the $x, y$ and the $z$ axes were introduced for simulations of the experimental PELDOR time traces.

spin is $1.15 \mathrm{~nm}$ for the ${ }^{\mathrm{Im}_{\mathrm{U}}} \mathrm{U}$ and ${ }^{\mathrm{Ox}} \mathrm{U}$ spin label and $1.95 \mathrm{~nm}$ for the ${ }^{\text {ExIm}} \mathrm{U}$ along this vector, the coordinates of the electron spin could be calculated. These distances and angles are estimated from the model shown in the ESI of Gophane et al. ${ }^{25}$ To allow for the intrinsic internal mobility of the spin labels, we introduced additional rotational motions around the spin label molecular axis. The first one is a rotation around the linker between the spin label and the nucleic acid base. This is calculated as a rotation around the $x$ axis of the spin label (Fig. 4). To model other residual flexibility of the spin label to describe the change of the spatial position of the electron spin relative to the DNA we introduces small angle fluctuation about the other two axis $(y$ and $z$ ). All rotations originate at the $\mathrm{C} 5$ atom of the uracil.

The $y$ rotation has a more pronounced effect to the damping of the PELDOR oscillation and was adapted to fit the experimental observed damping of the oscillations. The small angle fluctuations around the $z$-axis have similar effect to the damping but a factor of 10 less. Because it could not be fully distinguished between both fluctuations, the $z$-rotation were fixed to $\pm 5^{\circ}$ for all simulations. All three axis motions were calculated independently from each other and are based on Gaussian-distributed angle variations. The calculated electron spin coordinates and the coordinate system of the nitroxide were transformed to distances and Euler angles with respect to a laboratory frame in order to simulate the PELDOR time traces. All optimizations are made by visual comparison of the simulated and experimental time traces as well as by a least square fit procedure. Details regarding our time trace simulation procedure has previously been described. ${ }^{20}$ Due to poor reproducibility of the modulation depth at G-band, only the frequency of the oscillations and the damping was taken into account for these experiments. Variations in modulation depths can be caused by uncertainties of the resonator performance and calculation of the excitation profile.

\section{Result and discussion}

Fig. 5 shows the best simulation results obtained for the three spin labels ${ }^{{ }^{I m}} U$, ${ }^{\text {Ox}} U$ and ${ }^{\text {ExIm}} U$ for the DNA(1,9) construct (see Fig. S5 and S6, ESI $\dagger$ for the corresponding results for the $\operatorname{DNA}(1,12)$ construct). In contrast to the spin label Ç, all the three semi-rigid isoindoline spin labels show some intrinsic motion in addition to the mobility of the dsDNA molecule itself. The main internal motion of the spin labels was expected to be around the $x$-axis of the nitroxide molecular axis system. To simulate the other residual motions of the spin label with respect to the DNA molecular frame, small angle Gaussian distributed fluctuations around the $z$ - and $y$-axis have been included in our model. The respective angles for all three axis rotations are reported in Table 1.

The rotation around the $x$ axis is of special interest because it directly reflects the expected rotational flexibility around the $\mathrm{N}-\mathrm{O}$ bond of the spin label. Whereas this rotation is strongly hindered for ${ }^{{ }^{I m}} U$ and ${ }^{O x} U,{ }^{\text {ExIm}} U$ rotates freely around this bond (a $90^{\circ}$ rotation angle distribution reflects a full rotation due to symmetry reasons). For orientation selective measurements, a high rotational freedom is a disadvantage, as it leads to loss of orientation information. On the other hand, the distances from such data might be more easily predicted. Thus ${ }^{\text {ExIm }} U$ is an almost perfect spin label, for obtaining high-precision distance data alone, although possible structural perturbations in complex folds or in macromolecular complexes must be considered. In comparison the ${ }^{\mathrm{Im}} \mathrm{U}$ is limited to an $x$-rotation of $\pm 5^{\circ}$, whereas the ${ }^{\mathrm{Ox}} \mathrm{U}$ spin label has rotational freedom of $\pm 10^{\circ}$. These values are independent of the spin label position. Thus, the determined $x$-axis rotational freedom of the spin labels are in very good agreement with the expectations and fully support the influence of a hydrogen bond between the spin label and the nucleobase in the case of the ${ }^{\mathrm{Im}} \mathrm{U}$ spin label. The other two rotations reflect the overall residual flexibility of the spin label. These values should be as small as possible to allow a detailed study of the dynamic of the DNA molecule itself. They are rather similar for all three spin labels investigated, demonstrating that the main difference in the motion of the three spin labels can indeed be described by their rotational freedom around the $x$-axis. The rotation around the $y$ axis, which correspond to an up and down movement in direction of the helix axis, results for the isoindoline derived spin labels to rotation angles between $\pm 14^{\circ}$ to $\pm 22^{\circ}$. These values can be compared to the fluctuations found for the Ç spin label (Fig. S3, ESI $\dagger$ ). This rigid spin label shows a small fluctuation with $6^{\circ}$ for the $y$-rotation, while no $x$-rotation could be observed. These small vibration, could be interpreted as DNA dynamics, that are not explicitly described by our physical model.

These best fit parameters are slightly larger for ${ }^{\text {Ox }} \mathrm{U}$. Additionally these values are slightly larger for the $\operatorname{DNA}(1,12)$. This could by caused by a higher mobility at the end of the DNA strand and thus reflect also partly motion of the DNA itself. More systematic studies, including the rigid spin label Ç, have 




$\mathrm{B}$
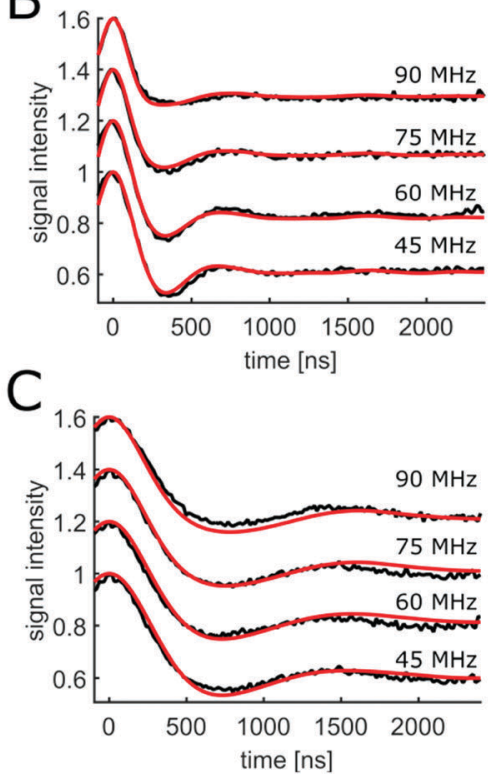

Q-band $(33 \mathrm{GHz})$
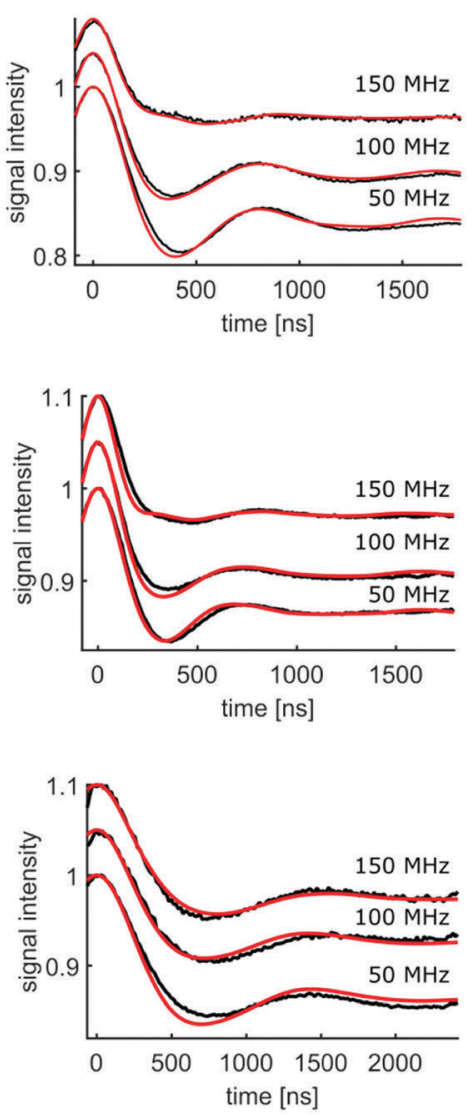

G-band $(180 \mathrm{GHz})$


Fig. 5 Experimental (black) and simulated (red) PELDOR time traces. For X-band (right) and Q-band (middle) different offsets $\Delta \nu$ (in $\mathrm{MHz}$ ) between probe and pump pulse frequency for DNA(1,9) with (A) ${ }^{I m} U(B){ }^{O x} U(C)$ ExlmU spin label. G-band (left) PELDOR time traces at the three magnetic field positions corresponding to the main $G$-tensor components. In these experiments, the difference between pump and probe frequency was kept constant at $\Delta \nu=60 \mathrm{MHz}$.

Table 1 Result for the flexibility of the different spin labels in respect to their position at the DNA. The values represent the result of the global analysis over all frequencies. The numbers in brackets show the error. An example for error estimation is given in the ESI

\begin{tabular}{llll}
\hline & $x$-rotation & $y$-rotation & $z$-rotation \\
\hline${ }^{\operatorname{Im}} \mathrm{U}-\mathrm{DNA}(1,9)$ & $5^{\circ}(2)$ & $14^{\circ}(1)$ & $5^{\circ}$ \\
${ }^{\mathrm{Im}} \mathrm{U}-\mathrm{DNA}(1,12)$ & $5^{\circ}(2)$ & $20^{\circ}(1)$ & $5^{\circ}$ \\
${ }^{\mathrm{Ox}} \mathrm{U}-\mathrm{DNA}(1,9)$ & $10^{\circ}(2)$ & $18^{\circ}(1)$ & $5^{\circ}$ \\
${ }^{\text {Ox }} \mathrm{U}-\mathrm{DNA}(1,12)$ & $10^{\circ}(5)$ & $22^{\circ}(2)$ & $5^{\circ}$ \\
${ }^{\text {ExIm }} \mathrm{U}-\mathrm{DNA}(1,9)$ & $90^{\circ}(5)$ & $12^{\circ}(2)$ & $5^{\circ}$ \\
${ }^{\text {ExIm }} \mathrm{U}-\mathrm{DNA}(1,12)$ & $90^{\circ}(10)$ & $16^{\circ}(2)$ & $5^{\circ}$
\end{tabular}

to be performed to investigate this further. Overall the spin label ${ }^{\operatorname{Im}} \mathrm{U}$ is somewhat better for orientation measurements, compared to the ${ }^{\mathrm{Ox}} \mathrm{U}$ spin label.

The simulations that were performed were based on simplified models of the overall conformational motion of the DNA molecule and the spin label and, therefore, the question arises about the uniqueness of the solution and the accurateness of the obtained values. All rotation parameters for the spin label movement were estimated by comparison of the simulated and experimental data from measurements at X- and Q-band.
The $y$-axis rotation is already rather well defined by the X-band data, because it strongly influences the damping of the dipolar oscillations. For the $x$-axis rotation only an accuracy of about $\pm 10^{\circ}$ was possible with the $\mathrm{X}$-band data alone. The effect of this rotation is mainly seen as a change in modulation depth of the $\mathrm{X}$-band time traces for large offsets between pump and probe pulses. However at Q-band frequencies, variation of the $x$-axis rotation shows also more sensitive changes in the modulation period of the time trace with $15 \mathrm{MHz}$ offset between pump and probe frequency. Despite the fact that these effects are quite small, it led to an enhanced precision of the determination of this important rotation angle to $\pm 3^{\circ}$. Additional experiments at G-band frequency were performed to take advantage of the fact that at high fields the anisotropy of the $g$ - and $A$-tensor is fully spectrally resolved, allowing one to distinguish all orientations with high precision. Simulations using the (low field) optimized parameters were performed and compared with the experimental data, as depicted in Fig. S4 in the ESI. $\dagger$ A large deviation between experiment and prediction was observed, especially at the $g_{x x}$ position. A small fixed rotation of the spin label of $9^{\circ}$ around the $x$ axis ( $\mathrm{N}-\mathrm{O}$ bond), before the rotation started, improves the fit without influencing the (low field) simulation 
time traces. This reflects a slight tilting of the spin label plane with respect to the nucleobase plane. With this very small readjustment of the spin label position, very good predictions of the G-band PELDOR time traces taken at the canonical $g$-tensor positions could be achieved, indicating that our model with the optimized parameters can indeed be used to accurately model the spin labels. This rotation was also applied to the analysis of the other two spin labels, since they have the same geometry.

\section{Conclusion}

In this work orientation selective PELDOR experiments were performed at multiple magnetic fields $(0.3 \mathrm{~T}, 1.2 \mathrm{~T}$ and $6.4 \mathrm{~T})$ and allowed the determination of the flexibility of the isoindoline-derived spin labels ${ }^{\text {ExIm }} U,{ }^{{ }^{I m}} U$ and ${ }^{O x} U$, all of which were covalently attached to a dsDNA. For this purpose, the earlier proposed model for the dsDNA dynamics, consisting of a negative correlated twist-stretch motion with a radius change of the $\mathrm{DNA}^{21,31}$ was extended to include the intrinsic rotational freedom of the spin labels. Simulations were found to be in very good agreement with the experimental time traces, which verified the expected different degree of rotational freedom around the $\mathrm{N}-\mathrm{O}$ axis for the conformationally unambiguous spin label ${ }^{\text {ExIm}_{U}} \mathrm{U}$ and the two semi-rigid spin labels, ${ }^{{ }^{\mathrm{m}}} \mathrm{U}$ and ${ }^{O x} U$. The spin labels showed some additional flexibility, which could be modeled by a $10-20^{\circ}$ rotation around the $y$-axis of the nitroxide molecular axis system. The dependence of this parameter on the position of the spin label might indicate some additional DNA dynamics at the ends of the helix. The parameters describing the internal flexibility of the spin labels, which were obtained from X-band and Q-band PELDOR experiments, predicted very accurately the high-field PELDOR time traces at G-band frequencies. Thus, we believe that our parameterization of the intrinsic nitroxide dynamics is reliable and can be used also for modeling of more complex DNA structures. That will allow to use these new spin labels to study the conformational flexibilities of nucleic acids with respect to their specific sequence, the ionic strength and other parameters effecting their structure and dynamics.

\section{Acknowledgements}

We acknowledge financial support from the German Research Foundation (CRC902: Molecular Principles of RNA Based Regulation) and the Icelandic Research Fund (120001022).

\section{References}

1 D. Klostermeier and C. Hammann, RNA Structure and Folding, Walter de Gruyter GmbH, 2013.

2 H. M. Al and N. G. Walter, Curr. Opin. Struct. Biol., 2008, 18, 321-329.

3 B. Fürtig, C. Richter, J. Wöhnert and H. Schwalbe, ChemBioChem, 2003, 4, 936-962.

4 J. Hennig and M. Sattler, Protein Sci., 2014, 23, 669-682.

5 A. Lapinaite, B. Simon, L. Skjaerven, M. Rakwalska-Bange, F. Gabel and T. Carlomagno, Nature, 2013, 502, 519-523.
6 A. Reining, S. Nozinovic, K. Schlepckow, F. Buhr, B. Fürtig and H. Schwalbe, Nature, 2013, 499, 355-359.

7 A. Iqbal, S. Arslan, B. Okumus, T. J. Wilson, G. Giraud, D. G. Norman, T. Ha and D. M. J. Lilley, Proc. Natl. Acad. Sci. U. S. A., 2008, 105, 11176-11181.

8 A. K. Wozniak, G. F. Schröder, H. Grubmüller, C. A. M. Seidel and F. Oesterhelt, Proc. Natl. Acad. Sci. U. S. A., 2008, 105, 18337-18342.

9 A. D. Milov, K. Salikhov and M. Shchirov, Sov. Phys. Solid State, 1981, 23, 975-982.

10 A. D. Milov, A. Ponomarev and Y. D. Tsvetkov, Lett. Chem. Phys., 1984, 110, 67-72.

11 M. Pannier, S. Veit, A. Godt, G. Jeschke and H. W. Spiess, J. Magn. Reson., 2011, 213, 316-325.

12 T. Prisner, M. Rohrer and F. MacMillan, Annu. Rev. Phys. Chem., 2001, 52, 279-313.

13 G. W. Reginsson, S. A. Shelke, C. Rouillon, M. F. White, S. T. Sigurdsson and O. Schiemann, Nucleic Acids Res., 2013, 41, 1-10.

14 D. Margraf, B. E. Bode, A. Marko, O. Schiemann and T. F. Prisner, Mol. Phys., 2007, 105, 2153-2160.

15 W. L. Hubbell, D. S. Cafiso and C. Altenbach, Nat. Struct. Biol., 2000, 7, 735-739.

16 S. A. Shelke and S. T. Sigurdsson, Angew. Chem., Int. Ed. Engl., 2010, 49, 7984-7986.

17 N. Barhate, P. Cekan, A. P. Massey and S. T. Sigurdsson, Angew. Chem., 2007, 119, 2709-2712.

18 C. Höbartner, G. Sicoli, F. Wachowius, D. B. Gophane and S. T. Sigurdsson, J. Org. Chem., 2012, 77, 7749-7754.

19 T. F. Prisner, a. Marko and S. T. Sigurdsson, J. Magn. Reson., 2015, 252, 187-198.

20 A. Marko, D. Margraf, P. Cekan, S. T. Sigurdsson, O. Schiemann and T. F. Prisner, Phys. Rev. E: Stat., Nonlinear, Soft Matter Phys., 2010, 81, 1-9.

21 A. Marko, V. Denysenkov, D. Margraf, P. Cekan, O. Schiemann, S. T. Sigurdsson and T. F. Prisner, J. Am. Chem. Soc., 2011, 133, 13375.

22 C. M. Grytz, A. Marko, P. Cekan, S. T. Sigurdsson and T. F. Prisner, Phys. Chem. Chem. Phys., 2016, 18, 2993-3002.

23 D. B. Gophane and S. T. Sigurdsson, Chem. Commun., 2013, 49, 999-1001.

24 M. Sajid, G. Jeschke, M. Wiebcke and A. Godt, Chem. - Eur. J., 2009, 15, 12960-12962.

25 D. B. Gophane, B. Endeward, T. F. Prisner and S. T. Sigurdsson, Chem. - Eur. J., 2014, 20, 15913-15919.

26 Y. Polyhach, E. Bordignon, R. Tschaggelar, S. Gandra, A. Godt and G. Jeschke, Phys. Chem. Chem. Phys., 2012, 14, 10762.

27 G. Jeschke, V. Chechik, P. Ionita, A. Godt, H. Zimmermann, J. Banham, C. R. Timmel, D. Hilger and H. Jung, Appl. Magn. Reson., 2006, 30, 473-498.

28 M. M. Hertel, V. P. Denysenkov, M. Bennati and T. F. Prisner, Magn. Reson. Chem., 2005, 43, S248-S255.

29 M. Rohrer, O. Brügmann, B. Kinzer and T. Prisner, Appl. Magn. Reson., 2001, 21, 257-274.

30 V. P. Denysenkov, T. F. Prisner, J. Stubbe and M. Bennati, Appl. Magn. Reson., 2005, 29, 375-384.

31 J. Gore, Z. Bryant, M. Nöllmann, M. U. Le, N. R. Cozzarelli and C. Bustamante, Nature, 2006, 442, 836-839. 